\title{
ERRATUM
}

\section{3-Methylglutaconyl-CoA hydratase from Acinetobacter sp}

\author{
Matthias Mack • Michael Liesert · Johannes Zschocke · \\ Verena Peters · Dietmar Linder · Wolfgang Buckel
}

Published online: 29 April 2006

(C) Springer-Verlag 2006

\section{Arch Microbiol (2006) DOI 10.1007/s00203-006-0095-7}

Unfortunately, it escaped our attention that the same enzyme, though from another organism, had been described already 2 years earlier.

Wong BJ, Gerlt JA (2004) Evolution of function in the crotonase superfamily: (3S)-methylglutaconyl-CoA hydratase from Pseudomonas putida. Biochemistry 43:4646-4654.

The online version of the original article can be found at http:// dx.doi.org/10.1007/s00203-006-0095-7

M. Mack $(\bowtie)$

Institut für Technische Mikrobiologie, Fachhochschule

Mannheim, Hochschule für Technik und Gestaltung,

Windeckstr. 110, 68163 Mannheim, Germany

e-mail: m.mack@hs-mannheim.de

M. Liesert · W. Buckel

Laboratorium für Mikrobiologie, Fachbereich Biologie,

Philipps-Universität, 35032 Marburg, Germany

J. Zschocke $\cdot$ V. Peters

Abteilung für Allgemeine Pädiatrie und Institut für

Humangenetik, Ruprecht-Karls-Universität, Heidelberg,

Germany

D. Linder

Institut für Biochemie, Justus-Liebig-Universität, Giessen,

Germany 